Proceedings of the Institute of Mathematics and Mechanics,

National Academy of Sciences of Azerbaijan

Volume 45, Number 2, 2019, Pages 319-330

https://doi.org/10.29228/proc. 12

\title{
SPECTRAL PROPERTIES FOR THE EQUATION OF VIBRATING BEAM WITH A SPECTRAL PARAMETER IN THE BOUNDARY CONDITIONS
}

\author{
VUQAR A. MEKHRABOV
}

\begin{abstract}
In this paper we consider the spectral problem for ordinary differential equation of fourth order with a spectral parameter in the boundary conditions. This problem arises when describing the bending vibrations of a homogeneous rod, in cross-sections of which the longitudinal force acts, the left end of which is rigidly fixed, and the inertial mass is concentrated on the right end and also at this end a tracking force acts. We study the location of eigenvalues on the real axis, find the multiplicities of all eigenvalues, examine the oscillation properties of eigenfunctions and establish sufficient conditions for the subsystems of root functions of this problem to form a basis in $L_{p}, 1<p<\infty$.
\end{abstract}

\section{Introduction}

We consider the following eigenvalue problem:

$$
\begin{gathered}
y^{(4)}(x)-\left(q(x) y^{\prime}(x)\right)^{\prime}=\lambda y(x), 0<x<1, \\
U_{1}(\lambda, y) \equiv y(0)=0, U_{2}(\lambda, y) \equiv y^{\prime}(0)=0, \\
U_{3}(\lambda, y) \equiv y^{\prime \prime}(1)-a_{1} \lambda y^{\prime}(1)=0 \\
U_{4}(\lambda, y) \equiv T y(1)-a_{2} \lambda y(1)=0
\end{gathered}
$$

where $\lambda \in \mathbb{C}$ is a spectral parameter, $T y \equiv y^{\prime \prime \prime}-q y^{\prime}, q(x)$ is a positive absolutely continuous function on the interval $[0,1], a_{1}, a_{2}$ are real constants such that $a_{1}<$ $0, a_{2}>0$.

Problem (1.1)-(1.4) arises when describing the bending vibrations of a homogeneous rod, in cross-sections of which the longitudinal force acts, the left end of which is rigidly fixed, and the inertial mass is concentrated on the right end and also at this end a tracking force acts (see, for example, [17, p. 152-154]).

The study of the spectral properties of ordinary differential operators with a spectral parameter in the boundary conditions has a long history. Problems of this type arise from the specific problems of mechanics and mathematical physics (see [17, 18, 20, 26, 29, 31-33] and others). For the Sturm-Liouville

2010 Mathematics Subject Classification. 34B05, 34B09, 34B24, 34L10, 34L15, 74H45 .

Key words and phrases. beam equation, spectral parameter, location of eigenvalues, basis property of root functions. 
problems with a spectral parameter in the boundary conditions, the spectral properties, including the basis properties of root functions in various functional spaces, are well studied (see $[3-6,15,16,18-20,25,27,30,33]$ ). The spectral properties of the boundary value problems for ordinary differential equations of fourth order with a spectral parameter in the boundary conditions were studied in $[1,2,7-11,14,21-24]$. The basis properties of systems of eigenfunctions in $L_{p}(0,1), 1<p<\infty$ of considered problems were studied in [1, 2, 8-11, 23]. In these works sufficient conditions were found for subsystems of eigenfunctions to form a basis in $L_{p}(0,1), 1<p<\infty$.

In [7, 9-11], boundary value problems for ordinary differential equations of fourth order with a spectral parameter contained in two of boundary conditions were considered. In $[7,10,11]$ the establishment of sufficient conditions for the systems of eigenfunctions of the problems under consideration after removing two functions to form a basis in the space $L_{p}(0,1), 1<p<\infty$ was based on the rough asymptotic formulas for eigenvalues and eigenfunctions, and the oscillation properties of eigenfunctions and their derivatives. In [9], this was based on finer asymptotic formulas for eigenvalues and eigenfunctions.

Note that signs of the parameters $a_{1}$ and $a_{2}$ play an important role. If $a_{1}>0$ and $a_{2}<0$, then problem (1.1)-(1.4) can be treated as a spectral problem for a self-adjoint operator in the Hilbert space $H=L_{2}(0,1) \oplus \mathbb{C}^{2}$. If $a_{1}>0$ and $a_{2}>0$, then this problem is equivalent to a spectral problem for the $J$-self-adjoint operator in the Pontryagin space $\Pi_{1}=L_{2}(0,1) \oplus \mathbb{C}^{2}$ with the corresponding inner product. If $a_{1}<0$ and $a_{2}>0$, then problem (1.1)-(1.4) is reduced to a spectral problem for the $J$-self-adjoint operator in the Pontryagin space $\Pi_{2}=L_{2}(0,1) \oplus \mathbb{C}^{2}$. In the case $a_{1}>0$ and $a_{2}<0$ all eigenvalues of problem (1.1)-(1.4) are positive, simple, and form an infinitely increasing sequence. In the case $a_{1}>0$ and $a_{2}>0$ problem (1.1)-(1.4) has one negative simple eigenvalue and a sequence of positive and simple eigenvalues tends to infinity [9]. In the case $a_{1}<0$ and $a_{2}>0$ we show that this problem has two negative simple eigenvalues and a sequence of positive and simple eigenvalues tends to infinity.

The purpose of the present paper is to study the location of eigenvalues on real axis, the structure of root subspaces and the basis properties of subsystems of eigenfunctions of problem (1.1)-(1.4), which is traditional in spectral theory.

\section{Preliminaries}

Along with problem (1.1)-(1.4) we consider the following spectral problem

$$
\begin{gathered}
y^{(4)}(x)-\left(q(x) y^{\prime}(x)\right)^{\prime}=\lambda y(x), 0<x<1, \\
y(0)=y^{\prime}(0)=0, \\
y^{\prime}(1) \cos \gamma+y^{\prime \prime}(1) \sin \gamma=0, \\
T y(1)-a_{2} \lambda y(1)=0,
\end{gathered}
$$

where $\gamma \in\left[0, \frac{\pi}{2}\right]$. A more general form of the problem (2.1) was considered in [2].

The following theorem is a special case of the central result of [2].

Theorem 2.1. The eigenvalues of the spectral problem (2.1) are real, simple and form an infinitely increasing sequence $\lambda_{1}(\gamma), \lambda_{2}(\gamma), \ldots, \lambda_{k}(\gamma), \ldots$ Moreover, for each $\gamma \in(0, \pi / 2)$ these eigenvalues have the following location on the real 
axis:

$$
\lambda_{1}(0)<\lambda_{1}(\gamma)<\lambda_{1}(\pi / 2)<0<\lambda_{2}(\pi / 2)<\lambda_{2}(\gamma)<\lambda_{2}(0)<\ldots
$$

We need the following result which is basic in the sequel.

Theorem 2.2. [9, Theorem 3.1] For each fixed $\lambda \in \mathbb{C}$ there exists a nontrivial solution $y(x, \lambda)$ of problem (1.1), (1.2), (1.4) which is unique up to a constant coefficient.

Remark 2.1. Without loss of generality we can assume that the function $y(x, \lambda)$ for each fixed $x \in[0,1]$ is as an entire function of $\lambda$.

Let $D_{k}=\left(\lambda_{k-1}(0), \lambda_{k}(0)\right), k=1,2, \ldots$, where $\lambda_{0}(0)=-\infty$.

Obviously, the eigenvalues $\lambda_{k}(0)$ and $\lambda_{k}(\pi / 2)$ of the spectral problem (2.1) for $\gamma=0$ and $\gamma=\pi / 2$ are zeros of entire functions $y^{\prime}(1, \lambda)$ and $y^{\prime \prime}(1, \lambda)$, respectively. We see that the function $G(\lambda)=\frac{y^{\prime \prime}(1, \lambda)}{y^{\prime}(1, \lambda)}$ is well defined for $\lambda \in D \equiv(\mathbb{C} \backslash \mathbb{R}) \cup$ $\left(\bigcup_{k=1}^{\infty} D_{k}\right)$. Moreover, $\lambda_{k}(\pi / 2)$ and $\lambda_{k}(0), k \in \mathbb{N}$, are zeros and poles of the function $G(\lambda)$, respectively.

Lemma 2.1. The following relations hold:

$$
\begin{gathered}
\frac{d G(\lambda)}{d \lambda}=-\frac{1}{y^{\prime 2}(1, \lambda)}\left\{\int_{0}^{l} y^{2}(x, \lambda) d x-a_{2} y^{2}(1, \lambda)\right\}, \lambda \in D . \\
\lim _{\lambda \rightarrow-\infty} G(\lambda)=+\infty .
\end{gathered}
$$

The proof of this lemma is similar to that of [7, Lemmas 3.3 and 3.4].

The following result directly follows from Theorems 2.1, 2.2, Remark 2.1 and Lemma 2.1.

Lemma 2.2. The following relations hold:

(i) $G(\lambda)>0$ for $\lambda \in\left(-\infty, \lambda_{1}(0)\right)$ and

$$
\lim _{\lambda \rightarrow \lambda_{1}(0)-0} G(\lambda)=+\infty
$$

(ii) $G(\lambda)<0$ for $\lambda \in\left(\lambda_{1}(0), \lambda_{1}(\pi / 2)\right)$,

$G(\lambda)>0$ for $\lambda \in\left(\lambda_{1}(\pi / 2), \lambda_{2}(\pi / 2)\right)$,

$G(\lambda)<0$ for $\lambda \in\left(\lambda_{2}(\pi / 2), \lambda_{2}(0)\right)$ and

$$
\lim _{\lambda \rightarrow \lambda_{1}(0)+0} G(\lambda)=\lim _{\lambda \rightarrow \lambda_{2}(0)-0} G(\lambda)=-\infty ;
$$

(iii) $G(\lambda)>0$ for $\lambda \in\left(\lambda_{k}(0), \lambda_{k+1}(\pi / 2)\right)$, $G(\lambda)<0$ for $\lambda \in\left(\lambda_{k+1}(\pi / 2), \lambda_{k+1}(0)\right)$ and

$$
\lim _{\lambda \rightarrow \lambda_{k}(0)+0} G(\lambda)=+\infty, \lim _{\lambda \rightarrow \lambda_{k+1}(0)-0} G(\lambda)=-\infty, k=2,3, \ldots .
$$

Lemma 2.3. [9, Lemma 3.3] The following representation holds:

$$
G(\lambda)=G(0)+\sum_{k=1}^{\infty} \frac{\lambda c_{k}}{\lambda_{k}(0)\left(\lambda-\lambda_{k}(0)\right)}, \lambda \in D,
$$


where $c_{k}=\underset{\lambda=\lambda_{k}(0)}{\operatorname{res}} G(\lambda)$, and $c_{1}<0, c_{k}>0$ for $k \geq 2$.

Lemma 2.4. $G(\lambda)$ is a concave function in the interval $\left(\lambda_{1}(0), \lambda_{2}(0)\right)$.

Proof. It follows from (2.5) that

$$
\frac{d^{2} G(\lambda)}{d \lambda^{2}}=-2 \sum_{k=1}^{\infty} \frac{c_{k}}{\left(\lambda-\lambda_{k}(0)\right)^{3}} .
$$

Hence from this relation we get

$$
\frac{d^{2} G(\lambda)}{d \lambda^{2}}>0 \text { for } \lambda \in\left(\lambda_{1}(0), \lambda_{2}(0)\right) .
$$

The proof of this lemma is complete.

\section{The structure of root subspaces of the boundary value problem (1.1)-(1.4)}

Lemma 3.1. The eigenvalues of the boundary value problem (1.1)-(1.4) are real, simple and form at most a countable set without a finite limit point.

Proof. It is obvious that the eigenvalues of problem (1.1)-(1.4) are roots of the equation

$$
y^{\prime \prime}(1, \lambda)-a_{1} \lambda y^{\prime}(1, \lambda)=0 .
$$

Let $\lambda$ be the nonreal eigenvalue of problem (1.1)-(1.4). Since the coefficients $q(x), a_{1}, a_{2}$ are real it follows that $\bar{\lambda}$ is also an eigenvalue of this problem and $y(x, \bar{\lambda})=\overline{y(x, \lambda)}$.

By virtue of $[1$, formula (3.6)] for each $\mu, \lambda \in \mathbb{C}$ we have

$$
\begin{gathered}
-y^{\prime \prime}(1, \mu) y^{\prime}(1, \lambda)+y^{\prime \prime}(1, \lambda) y^{\prime}(1, \mu)= \\
(\mu-\lambda)\left\{\int_{0}^{1} y(x, \mu) y(x, \lambda) d x-a_{2} y(1, \mu) y(1, \lambda)\right\} .
\end{gathered}
$$

Putting $\mu=\bar{\lambda}$ in (3.2) we obtain

$$
\begin{aligned}
& -\overline{y^{\prime \prime}(1, \lambda)} y^{\prime}(1, \lambda)+y^{\prime \prime}(1, \lambda) \overline{y^{\prime}(1, \lambda)}= \\
& (\bar{\lambda}-\lambda)\left\{\int_{0}^{1}|y(x, \lambda)|^{2} d x-a_{2}|y(1, \lambda)|^{2}\right\} .
\end{aligned}
$$

By virtue of (3.1) from (3.3) we get

$$
-a_{1}(\bar{\lambda}-\lambda)\left|y^{\prime}(1, \lambda)\right|^{2}=(\bar{\lambda}-\lambda)\left\{\int_{0}^{1}|y(x, \lambda)|^{2} d x-a_{2}|y(1, \lambda)|^{2}\right\},
$$

which implies that

$$
\int_{0}^{1}|y(x, \lambda)|^{2} d x+a_{1}\left|y^{\prime}(1, \lambda)\right|^{2}-a_{2}|y(1, \lambda)|^{2}=0,
$$

in view of the condition $\bar{\lambda} \neq \lambda$.

On the other hand multiplying both sides of equation (1.1) by $\overline{y(x, \lambda)}$, integrating the resulting equality in the range from 0 to 1 , using the formula for 
integration by parts, and by taking into account the boundary conditions (1.2) and (1.4), we obtain

$$
\begin{gathered}
\int_{0}^{1}\left|y^{\prime \prime}(x, \lambda)\right|^{2} d x+\int_{0}^{1} q(x)\left|y^{\prime}(x, \lambda)\right|^{2} d x= \\
\lambda\left\{\int_{0}^{1}|y(x, \lambda)|^{2} d x+a_{1}\left|y^{\prime}(1, \lambda)\right|^{2}-a_{2}|y(1, \lambda)|^{2}\right\} .
\end{gathered}
$$

It follows from (3.4) and (3.5) that

$$
\int_{0}^{1}\left|y^{\prime \prime}(x, \lambda)\right|^{2} d x+\int_{0}^{1} q(x)\left|y^{\prime}(x, \lambda)\right|^{2} d x=0,
$$

which is impossible in view of condition (1.2). Hence the eigenvalues of problem (1.1)-(1.4) are real.

The entire function occurring on the left-hand side in Eq. (3.1) does not vanish for nonreal $\lambda$. Hence, it does not vanish identically. Consequently, its zeros form at most a countable set without a finite limit point.

Remark 3.1. If $\lambda$ is an eigenvalue of problem (1.1)-(1.4), then it follows from (3.1) and $(2.2)$ that $y^{\prime}(1, \lambda) \neq 0$. Consequently, each root of Eq. (3.1) (given its multiplicity) is also root of the equation

$$
G(\lambda)=a_{1} \lambda .
$$

To prove the simplicity of eigenvalues of problem (1.1)-(1.4), by virtue of Remark 3.1, it suffices to show that Eq. (3.6) has only simple roots.

If $\lambda=\tilde{\lambda}$ is a multiple root of Eq. (3.6), then we have

$$
G(\tilde{\lambda})=a_{1} \tilde{\lambda} \text { and } G^{\prime}(\tilde{\lambda})=a_{1} .
$$

Hence it follows from (2.3) that

$$
-\frac{1}{y^{\prime 2}(1, \tilde{\lambda})}\left\{\int_{0}^{l} y^{2}(x, \tilde{\lambda}) d x-a_{2} y^{2}(1, \tilde{\lambda})\right\}=a_{1},
$$

which implies that

$$
\int_{0}^{1} y^{2}(x, \tilde{\lambda}) d x-a_{2} y^{2}(1, \tilde{\lambda})+a_{1} y^{\prime 2}(1, \tilde{\lambda})=0 .
$$

Since all eigenvalues of problem (1.1)-(1.4) are real it follows from (3.5) and (3.8) that

$$
\int_{0}^{1} y^{\prime \prime 2}(x, \tilde{\lambda}) d x+\int_{0}^{1} q(x) y^{\prime 2}(x, \tilde{\lambda}) d x=0 .
$$

By (1.2) from the last relation we get $y(x, \tilde{\lambda}) \equiv 0$ which contradicts the condition $y(x, \tilde{\lambda}) \not \equiv 0$. The proof of this lemma is complete.

Lemma 3.2. For each $k \in \mathbb{N}, k \neq 2$, the eigenvalue problem (1.1)-(1.4) can have only one eigenvalue in the interval $D_{k}$. 
Proof. Let $\tilde{\lambda} \in D_{1}$ be an eigenvalue of problem (1.1)-(1.4), i.e. $G(\tilde{\lambda})-a_{1} \tilde{\lambda}=0$. Since $\tilde{\lambda}<0$ it follows from (3.5) that

$$
\int_{0}^{1} y^{2}(x, \tilde{\lambda}) d x-a_{2} y^{2}(1, \tilde{\lambda})+a_{1} y^{\prime 2}(1, \tilde{\lambda})<0 .
$$

By Remark 3.1, we can rewrite this relation in the following form

$$
-\frac{\int_{0}^{1} y^{2}(x, \tilde{\lambda}) d x-a_{2} y^{2}(1, \tilde{\lambda})}{y^{\prime 2}(1, \tilde{\lambda})}-a_{1}>0 .
$$

Hence it follows from (2.3) that

$$
G^{\prime}(\tilde{\lambda})-a_{1}>0 .
$$

Therefore, by virtue of (3.9), Eq. (3.6) has a unique solution $\tilde{\lambda}$ in $D_{1}$.

The assertion of this lemma for $k=3,4, \ldots$, is proved in a similar way. The proof of lemma is complete.

Theorem 3.1. There exists an unboundedly increasing sequence $\left\{\lambda_{k}\right\}_{k=1}^{\infty}$ of eigenvalues of the boundary value problem (1.1)-(1.4) such that

$$
\begin{aligned}
& \left.\lambda_{1} \in\left(-\infty, \lambda_{1}(0)\right), \lambda_{2} \in\left(\lambda_{1}(\pi / 2), 0\right)\right), \\
& \lambda_{k} \in\left(\lambda_{k-1}(\pi / 2), \lambda_{k-1}(0)\right), k=3,4, \ldots .
\end{aligned}
$$

Proof. By virtue of Lemma 2.4 we have $G(\lambda)>0$ for $\lambda \in D_{1}$. Moreover, by following the arguments in Lemma 3.4 of [7] one can justify that

$$
G(\lambda)=\sqrt{2} \sqrt[4]{|\lambda|}\left(1+O\left(\frac{1}{\sqrt[4]{|\lambda|}}\right)\right) \text { as } \lambda \rightarrow-\infty .
$$

By (3.11) and the condition $a_{1}<0$ it follows from Lemma 3.2 that Eq. (3.6) has only one solution $\lambda_{1}$ in the interval $D_{1}$.

In view of Lemma 2.3 the function $G(\lambda)$ is concave in the interval $D_{2}$. Moreover, by Lemma 2.4 we have $G(\lambda)<0$ for $\lambda \in\left(\lambda_{1}(0), \lambda_{1}(\pi / 2)\right), G(\lambda)>0$ for $\lambda \in\left(\lambda_{1}(\pi / 2), \lambda_{2}(\pi / 2)\right), G(\lambda)<0$ for $\lambda \in\left(\lambda_{2}(\pi / 2), \lambda_{2}(0)\right)$ and

$$
\lim _{\lambda \rightarrow \lambda_{1}(0)+0} G(\lambda)=\lim _{\lambda \rightarrow \lambda_{2}(0)-0} G(\lambda)=-\infty ;
$$

Hence it follows from Lemma 3.1 that Eq. (3.6) has two simple roots $\lambda_{2} \in$ $\left(\lambda_{1}(\pi / 2), 0\right)$ and $\lambda_{3} \in\left(\lambda_{2}(\pi / 2), \lambda_{2}(0)\right)$.

Since the function $G(\lambda)$ is continuous in $D_{k}, k \in \mathbb{N}$, it follows from assertion (iii) of Lemma 2.4 that this function takes each value in $(-\infty,+\infty)$ at some point in the interval $D_{k}, k=3,4, \ldots$. Then by Lemma $3.2 \mathrm{Eq}$. (3.6) has only one simple root $\lambda_{k+1}$ in each interval $D_{k}, k=3,4, \ldots$. Moreover, since $a_{1}<0$ and the function $G(\lambda)$ takes the value 0 in a unique point $\lambda_{k}(\pi / 2), k=3,4, \ldots$, it follows that $\left.\lambda_{k+1} \in\left(\lambda_{k}(\pi / 2)\right), \lambda_{k}(0)\right)$ for $k=3,4, \ldots$. The proof of this theorem is complete.

Theorem 3.2. One has the asymptotic formulas

$$
\sqrt[4]{\lambda_{k}}=(k-3 / 2) \pi+O(1 / k)
$$




$$
\begin{gathered}
y_{k}(x)=\sin (k-3 / 2) \pi x-\cos (k-3 / 2) \pi x+e^{-(k-3 / 2) \pi x}+ \\
+(-1)^{k+1} e^{-(k-3 / 2) \pi(1-x)}+O(1 / k),
\end{gathered}
$$

where relation (3.13) holds uniformly for $x \in[0,1]$.

The proof of this theorem is similar to that of [2, Theorem 5.1] with the use of Theorem 3.1.

\section{Basis properties of subsystems of eigenfunctions of the boundary value problem (1.1)-(1.4)}

The eigenvalue problem (1.1)-(1.4) can be reduced to the eigenvalue problem for the linear operator $L$ in the Hilbert space $H=L_{2}(0,1) \oplus \mathbb{C}^{2}$ with the inner product

$$
(\hat{u}, \hat{v})=(\{y, m, n\},\{v, s, t\})=(y, v)_{L_{2}}+a_{1}^{-1} m \bar{s}+a_{2}^{-1} n \bar{t}
$$

where

$$
L \hat{y}=L\{y, m, n\}=\left\{(T y(x))^{\prime}, y^{\prime \prime}(1), T y(1)\right\}
$$

is an operator with the domain

$$
\begin{gathered}
D(L)=\left\{\{y(x), m, n\}: y \in W_{2}^{4}(0,1),(T y(x))^{\prime} \in L_{2}(0,1),\right. \\
\left.y(0)=y^{\prime}(0)=0, m=a_{1} y^{\prime}(1), n=a_{2} y(1)\right\}
\end{gathered}
$$

dense everywhere in $H . L$ is a closed operator in $H$ with compact resolvent. The eigenvalue problem for the operator $L$ is adequate to problem (1.1)-(1.4), i.e. the spectra of operator $L$ and problem (1.1)-(1.4) coincide, as do their multiplicities; between the eigenvectors of operator $L$ and the eigenfunctions of problem (1.1)(1.4), there is a one-to-one correspondence

$$
y_{k}(x) \leftrightarrow\left\{y_{k}(x), m_{k}, n_{k}\right\}, m_{k}=a_{1} y_{k}^{\prime}(1), n_{k}=a_{2} y_{k}(1) .
$$

Since $a_{1}<0$ and $a_{2}>0, L$ is a nonself-adjoint operator in $H$. Then in this case we define an operator $J: H \rightarrow H$ as follows:

$$
J\{y, m ; n\}=\{y,-m,-n\} .
$$

$J$ is unitary and symmetric operator in $H$. Its spectrum consists of two eigenvalues: -1 with multiplicity 2 and +1 with infinite multiplicity. This operator generates the Pontryagin space $\Pi_{2}=L_{2}(0,1) \oplus \mathbb{C}^{2}$ with inner product [12]

$$
[\hat{u}, \hat{v}]=(\hat{u}, \hat{v})_{\Pi_{2}}=(\{y, m, n\},\{u, s, t\})_{\Pi_{2}}=(u, v)_{L_{2}}+a_{1}^{-1} m \bar{s}-a_{2}^{-1} n \bar{t},
$$

Theorem 4.1. The operator $L$ is $J$-self-adjoint in $\Pi_{2}$.

Proof. By virtue [16, Theorem 2.2] $J L$ is self-adjoint in $H$. Then, it follows from [13, Section 3, Corollary to Theorem 3.2] that operator $L$ is $J$-self-adjoint in $\Pi_{2}$.

Theorem 4.2. Let $L^{*}$ be the adjoint operator of $L$ in $H$. Then $L^{*}=J L J$. Moreover, the system of eigenvectors $\left\{\hat{y}_{k}\right\}_{k=1}^{\infty}, \hat{y}_{k}=\left\{y_{k}, m_{k}, n_{k}\right\}$, of $L$ forms a Riesz basis (after normalization) in $H$.

Proof. The proof of the first part of this theorem follows from [13, Section 3, Propostion $\left.5^{0}\right]$, and the second part from [13, Section 4, Theorem 4.2]. 
Since $\hat{y}_{k}=\left\{y_{k}, m_{k}, n_{k}\right\}, m_{k}=a_{1} y_{k}^{\prime}(1), n_{k}=a_{2} y_{k}(1), k \in \mathbb{N}$, is an eigenvector corresponding to the eigenvalue $\lambda_{k}$ of the operator $L$ it follows that

$$
L \hat{y}_{k}=\lambda_{k} \hat{y}_{k}, k \in \mathbb{N} \text {. }
$$

If $\left\{\hat{v}_{k}^{*}\right\}_{k=1}^{\infty}, \hat{v}_{k}^{*}=\left\{v_{k}^{*}, s_{k}^{*}, t_{k}^{*}\right\}$, is the system of eigenvectors of the operator $L^{*}$, then we have

$$
L^{*} \hat{v}_{k}^{*}=\lambda_{k} \hat{v}_{k}^{*}, k \in \mathbb{N} .
$$

Lemma 4.1. Elements of the system $\left\{\hat{v}_{k}\right\}_{k=1}^{\infty}, \hat{v}_{k}=\left\{v_{k}, s_{k}, t_{k}\right\}$, which is adjoint to the system $\left\{\hat{y}_{k}\right\}_{k=1}^{\infty}$, are given by the formula

$$
\hat{v}_{k}=\delta_{k}^{-1} \hat{y}_{k}, k \in \mathbb{N},
$$

where $\delta_{k}=\left[\hat{y}_{k}, \hat{y}_{k}\right], k \in \mathbb{N}$.

Proof. It follows from Theorem 4.2 and relations (4.1)-(4.4) that

$$
\hat{v}_{k}^{*}=J \hat{y}_{k}, k \in \mathbb{N} \text {. }
$$

By virtue of Theorem 4.1 the operator $L$ is $J$ - self-adjoint in the Pontryagin space $\Pi_{2}$. Hence the eigenvectors $\hat{y}_{k}$ and $\hat{y}_{l}, k \neq l$, of this operator corresponding to the eigenvalues $\lambda_{k}$ and $\lambda_{l}$ are $J$-orthogonal in $\Pi_{2}$. Then by (4.2) we have

$$
\left[\hat{y}_{k}, \hat{y}_{l}\right]=0 \text {. }
$$

It follows from Lemma 3.1 that for each $k \in \mathbb{N}$ the eigenvalue $\lambda_{k}$ of the operator $L$ is simple. Then by virtue of formula (3.6) we get

$$
G^{\prime}\left(\lambda_{k}\right)-a_{1} \neq 0 \text {. }
$$

Hence by (2.3) it follows from (4.8) that

$$
\left\|y_{k}\right\|_{L_{2}}^{2}+a_{1} y_{k}^{\prime 2}(1)-a_{2} y_{k}^{2}(1) \neq 0
$$

which implies that

$$
\left[\hat{y}_{k}, \hat{y}_{k}\right]=\left\|y_{k}\right\|_{L_{2}}^{2}+a_{1} y_{k}^{\prime 2}(1)-a_{2} y_{k}^{2}(1) \neq 0,
$$

in view of (4.2). Thus the formula (4.5) directly follows from relations (4.2), (4.6), (4.7) and (4.9). The proof of this lemma is complete.

We define numbers $\Delta_{r, l}, r, l \in \mathbb{N}$, as follows:

$$
\Delta_{r, l}=\left|\begin{array}{cc}
s_{r} & s_{l} \\
t_{r} & t_{l}
\end{array}\right|
$$

Theorem 4.3. Let $r$ and $l(r \neq l)$ be arbitrary fixed natural numbers. If $\Delta_{r, l} \neq 0$, then the system of eigenfunctions $\left\{y_{k}(x)\right\}_{k=1, k \neq r, l}^{\infty}$ of the spectral problem (1.1)(1.4) forms a basis in the space $L_{p}(0,1), 1<p<\infty$, which is an unconditional basis in the space $L_{2}(0,1)$; if $\Delta_{r, l}=0$, then this system is incomplete and nonminimal in $L_{p}(0,1), 1<p<\infty$.

The proof of Theorem 4.3 for $p=2$ is similar to that of [5, Theorem 4.1] with the use of Theorem 4.2 and relation $(4.5)$, for $p \in(1, \infty)$ and $p \neq 2$ is similar to that of [23, Theorem 5.1] with the use of formulas (3.12), (3.13).

For brevity, we introduce the notation $\sigma_{r, l}=a_{1} a_{2} \delta_{r}^{-1} \delta_{l}^{-1} y_{r}^{\prime}(1) y_{l}^{\prime}(1)$. Then, by Remark 3.1 and relation (4.8) it follows from (4.9) that $\sigma_{r, l} \neq 0$ and

$$
\Delta_{r, l}=\delta_{r}^{-1} \delta_{l}^{-1}\left|\begin{array}{cc}
m_{r} & m_{l} \\
n_{r} & n_{l}
\end{array}\right|=\sigma_{r, l}\left\{\frac{y_{r}(1)}{y_{r}^{\prime}(1)}-\frac{y_{l}(1)}{y_{l}^{\prime}(1)}\right\} .
$$


Then by (4.11) we have

$$
\Delta_{r, l}=\sigma_{r, l} \tilde{\Delta}_{r, l}
$$

where

$$
\tilde{\Delta}_{r, l}=\left\{\frac{y_{r}(1)}{y_{r}^{\prime}(1)}-\frac{y_{l}(1)}{y_{l}^{\prime}(1)}\right\}
$$

It follows from the proof of [5, Theorem 4.1] that the system

$$
\left\{u_{k}(x)\right\}_{k=1, k \neq r, l}^{\infty}, \quad u_{k}(x)=\left\{v_{k}(x)-\Delta_{r, l}^{-1}\left\{\Delta_{k, l} v_{r}(x)+\Delta_{r, k} v_{l}(x)\right\}\right\},
$$

is adjoint to the system $\left\{y_{k}(x)\right\}_{k=1, k \neq r, l}^{\infty}$.

Remark 4.1. By virtue of Theorem 4.3 and the relation (4.12) the fulfillment of the condition $\tilde{\Delta}_{r, l} \neq 0$ is necessary and sufficient for the system $\left\{y_{k}(x)\right\}_{k=1, k \text { ner }, l}^{\infty}$ to form a basis in the space $L_{p}(0,1), 1<p<\infty$.

Remark 4.2. It should be noted that, using the asymptotic formulas (3.12) and $(3.13)$, it is impossible to find a condition under which the relation $\tilde{\Delta}_{r, l} \neq$ 0 is satisfied. Therefore, in order to establish the conditions under which the system of eigenfunctions $\left\{y_{k}(x)\right\}_{k=1, k \neq r, l}^{\infty}$ of problem (1.1)-(1.4) forms a basis in $L_{p}(0,1), 1<p<\infty$, we need to obtain the asymptotic formula for the relation $\frac{y_{k}(1)}{y_{k}^{\prime}(1)}$ up to the term with $\frac{1}{k^{2}}$.

Since all the eigenvalues of problem (1.1)-(1.4) are positive, except the first two eigenvalues, in equation (1.1) we set $\lambda=\rho^{4}$ with $\rho>0$. As is known (see [28, Ch. II, $\S 4.5$ Theorem 1 and $\S 4.6$ formula (27)-(29)]), equation (1.1) has four linearly independent solutions $z_{k}(x)=z_{k}(x, \rho), k=1,2,3,4$, regular in $\rho$ (for sufficiently large $\rho>0$ ) and satisfying the relations

$$
z_{k}^{(s)}(x, \rho)=\left(\rho \omega_{k}\right)^{s} e^{\rho \omega_{k} x}\left\{1+\frac{q_{0}(x)}{4 \rho \omega_{k}}+O\left(\frac{1}{\rho^{2}}\right)\right\}, k=\overline{1,4}, k=\overline{0,3},
$$

where $q_{0}(x)=\int_{0}^{x} q(t) d t, \omega_{k}, k=1,2,3,4$, are the distinct 4 th roots of unity. We can assume that $\omega_{1}=-\omega_{4}=-1, \omega_{2}=-\omega_{3}=-i$.

By virtue of (1.2)-(1.4) we get

$$
\begin{aligned}
& U_{1}\left(z_{k}, \rho\right)=z_{k}(0, \rho), U_{2}\left(z_{k}, \rho\right)=z_{k}^{\prime}(0, \rho), \\
& U_{3}\left(z_{k}, \rho\right)=z_{k}^{\prime \prime}(1, \rho)-a_{1} z_{k}^{\prime}(1, \rho) \\
& U_{4}\left(z_{k}, \rho\right)=z_{k}^{\prime \prime \prime}(1, \rho)-q(1) z_{k}^{\prime}(1, \rho)-a_{2} \rho^{4} z_{k}(1, \rho) .
\end{aligned}
$$

It is obvious that the eigenfunction $y(x)=y(x, \rho)$ corresponding to the eigenvalue $\lambda=\rho^{4}$ of problem (1.2)-(1.4) can be represented in the form

$$
y(x, \rho)=\left|\begin{array}{cccc}
U_{1}\left(z_{1}, \rho\right) & U_{1}\left(z_{2}, \rho\right) & U_{1}\left(y_{3}, \rho\right) & U_{1}\left(z_{4}, \rho\right) \\
z_{1}(x) & z_{2}(x) & z_{3}(x) & z_{4}(x) \\
U_{3}\left(z_{1}, \rho\right) & U_{3}\left(z_{2}, \rho\right) & U_{3}\left(z_{3}, \rho\right) & U_{3}\left(z_{4}, \rho\right) \\
U_{4}\left(z_{1}, \rho\right) & U_{4}\left(z_{2}, \rho\right) & U_{4}\left(z_{3}, \rho\right) & U_{4}\left(z_{4}, \rho\right)
\end{array}\right|,
$$

which implies that

$$
y^{\prime}(x, \rho)=\left|\begin{array}{cccc}
U_{1}\left(z_{1}, \rho\right) & U_{1}\left(z_{2}, \rho\right) & U_{1}\left(y_{3}, \rho\right) & U_{1}\left(z_{4}, \rho\right) \\
z_{1}^{\prime}(x) & z_{2}^{\prime}(x) & z_{3}^{\prime}(x) & z_{4}^{\prime}(x) \\
U_{3}\left(z_{1}, \rho\right) & U_{3}\left(z_{2}, \rho\right) & U_{3}\left(z_{3}, \rho\right) & U_{3}\left(z_{4}, \rho\right) \\
U_{4}\left(z_{1}, \rho\right) & U_{4}\left(z_{2}, \rho\right) & U_{4}\left(z_{3}, \rho\right) & U_{4}\left(z_{4}, \rho\right)
\end{array}\right| .
$$


By following the arguments in [9, Theorem 5.4] and using (4.13)-(4.16), one can justify the following asymptotic formulas

$$
\begin{gathered}
y(1)=-4 a_{1} i \rho^{8} e^{\rho}\left(1+\frac{q_{0}}{4 \rho}\right)\left(1+O\left(\rho^{-2}\right)\right), \\
y^{\prime}(1)=-4 a_{2} i \rho^{7} e^{\rho}\left(1+\frac{q_{0}}{4 \rho}\right)\left\{1-\frac{1}{a_{2} \rho}+O\left(\rho^{-2}\right)\right\} .
\end{gathered}
$$

where $q_{0}=\int_{0}^{1} q(x) d x$. Hence it follows from the last relations that

$$
\frac{y_{k}(1)}{y_{k}^{\prime}(1)}=\frac{a_{1}}{a_{2}} \rho_{k}\left(1+\frac{1}{a_{2} \rho_{k}}+O\left(\frac{1}{\rho_{k}^{2}}\right)\right) \text {, }
$$

where $\rho_{k}=\sqrt[4]{\lambda_{k}}$.

Theorem 4.4. One has the following assertions:

(i) there exists $k_{0} \in \mathbb{N}$ such that for any $r, l>k_{0}(r>l)$ the system of eigenfunctions $\left\{y_{k}(x)\right\}_{k=1, k \neq r, l}^{\infty}$ of problem (1.1)-(1.4) forms a basis in $L_{p}(0,1), 1<$ $p<\infty$, which is an unconditional basis in $L_{2}(0,1)$;

(ii) for each fixed $l \in \mathbb{N}$ there exists $k_{l} \in \mathbb{N}$ such that for any $r>k_{l}$ the system of eigenfunctions $\left\{y_{k}(x)\right\}_{k=1, k \neq r, l}^{\infty}$ of problem (1.1)-(1.4) forms a basis in $L_{p}(0,1), 1<p<\infty$, which is an unconditional basis in $L_{2}(0,1)$.

Proof. It follows from (4.17) and (3.12) that there exists a natural number $k_{0}$ such that for any $r, l>k_{0}, r>l$, we have

$$
\begin{gathered}
\tilde{\Delta}_{r, l}=\frac{y_{r}(1)}{y_{r}^{\prime}(1)}-\frac{y_{l}(1)}{y_{l}^{\prime}(1)}=\frac{a_{1}}{a_{2}} \rho_{r}\left(1+\frac{1}{a_{2} \rho_{r}}+O\left(\frac{1}{\rho_{r}^{2}}\right)\right)- \\
\frac{a_{1}}{a_{2}} \rho_{l}\left(1+\frac{1}{a_{2} \rho_{l}}+O\left(\frac{1}{\rho_{l}^{2}}\right)\right)=\frac{a_{1}}{a_{2}}\left(\rho_{r}-\rho_{l}+O\left(\frac{1}{r}\right)+O\left(\frac{1}{l}\right)\right)= \\
\frac{a_{1}}{a_{2}}\left\{(r-3 / 2) \pi-(l-3 / 2) \pi+O\left(\frac{1}{r}\right)+O\left(\frac{1}{l}\right)\right\}> \\
\frac{a_{1}}{a_{2}}\left((r-l) \pi-\frac{M}{r}-\frac{M}{l}\right)>0,
\end{gathered}
$$

where $M>0$ is some constant. Then the statement (i) of this theorem follows from the last relation in view of Remark 4.1.

By (4.18) we have

$$
\Delta_{r, l}=\frac{a_{1}}{a_{2}}\left(\rho_{r}-\rho_{l}+O\left(\frac{1}{\rho_{r}}\right)+O\left(\frac{1}{\rho_{l}}\right)\right) .
$$

Hence it follows from (3.12) that for each $l \in \mathbb{N}$ there exists a sufficiently large $k_{l} \in \mathbb{N}$ such that

$$
\rho_{k_{l}}-\rho_{l}+O\left(\frac{1}{\rho_{r}}\right)+O\left(\frac{1}{\rho_{l}}\right)>0 .
$$

Then it follows that for any $r>k_{l}$ the relation $\Delta_{r, l}>0$ holds. Hence, by Remark 4.1, for each fixed $l \in \mathbb{N}$ and any $r>k_{l}$ the system of eigenfunctions $\left\{y_{k}(x)\right\}_{k=1, k \neq r, l}^{\infty}$ of problem (1.1)-(1.4) forms a basis in $L_{p}(0,1), 1<p<\infty$, which is an unconditional basis in $L_{2}(0,1)$. The proof of this theorem is complete. 


\section{References}

[1] Z.S. Aliyev, Basis properties of a fourth order differential operator with spectral parameter in the boundary condition, Cent. Eur. J. Math. 8 (2010), no. 2, 378-388.

[2] Z.S. Aliyev, Basis properties in $L_{p}$ of systems of root functions of a spectral problem with spectral parameter in a boundary condition, Diff. Equ. 47 (2011), no. 6, 766-777.

[3] Z.S. Aliyev, On basis properties of root functions of a boundary value problem containing a spectral parameter in the boundary conditions, Doklady Math. 87 (2013), no. 2, 137-139.

[4] Z.S. Aliyev, F.I. Allahverdi-zada, Some spectral properties of the boundary value problem with spectral parameter in the boundary conditions, Proc. Inst. Math. Mech.,Nat. Acad. Scien. Azerbaijan 40 (2014), no. 2, 52-64.

[5] Z.S. Aliyev, A.A. Dunyamaliyeva, Defect basis property of a system of root functions of a Sturm-Liouville problem with spectral parameter in the boundary conditions, Diff. Equ. 51 (2015), no. 10, 1249-1266.

[6] Z.S. Aliyev, A.A. Dunyamaliyeva, Ya.T. Mehraliyev, Basis properties in $L_{p}$ of root functions of Sturm-Liouville problem with spectral parameter-dependent boundary conditions, Mediterr. J. Math. 14 (2017), no. 3, 1-23.

[7] Z.S. Aliyev, S.B. Guliyeva, Properties of natural frequencies and harmonic bending vibrations of a rod at one end of which is concentrated inertial load, J. Differential Equations 263 (2017), no. 9, 5830-5845.

[8] Z.S. Aliyev, N.B. Kerimov, Spectral properties of the differential operators of the fourth-order with eigenvalue parameter dependent boundary condition, Int. J. Math. Math. Sci. 2012 (2012), Article ID 456517, 28 p.

[9] Z.S. Aliyev, N.B. Kerimov, V.A. Mekhrabov, On the convergence of expansions in eigenfunctions of a certain boundary value problem with a spectral parameter in the boundary conditions, I, II, Diff. Equ., to appear.

[10] Z.S. Aliyev Z.S., F.M. Namazov, Spectral properties of a fourth-order eigenvalue problem with spectral parameter in the boundary conditions, Elect. J. Diff. Equ. 2017 (2017), no. 307, 1-11.

[11] Z.S. Aliyev, F.M. Namazov, On the spectral problem arising in the mathematical model of bending vibrations of a homogeneous rod, Complex Anal. Oper. Theory, to appear, doi.org/10.1007/s11785-019-00924-z.

[12] T.Ya. Azizov, I.S. Iokhvidov, Linear operators in spaces with an indefinite metric, John Wiley, Chichester, UK, 1989

[13] T.Ya. Azizov, I.S. Iokhvidov, Linear operators in Hilbert spaces with $G$-metric, Uspekhi Mat. Nauk 26 (1971), no. 4, 43-92 (in Russian).

[14] J. Ben Amara, A.A. Vladimirov, On oscillation of eigenfunctions of a fourth-order problem with spectral parameters in the boundary conditions, J. Math. Sci. 150 (2008), no. 5, 2317-2325.

[15] P.A. Binding, P.J. Browne, K. Seddici, Sturm-Liouville problems with eigenparameter dependent boundary conditions, Proc. Edinburgh Math. Soc. 37 (1994), no. 1, 57-72.

[16] P.A. Binding, P.J. Browne, Application of two parameter eigencurves to SturmLiouville problems with eigenparameter dependent boundary conditions, Proc. Roy. Soc. Edinburgh, Sect. A: Math. 125 (1995), no. 6, 1205-1218.

[17] B.B. Bolotin, Vibrations in technique: Handbook in 6 volumes, The vibrations of linear systems, I, Engineering Industry, Moscow, 1978 (in Russian).

[18] C.T. Fulton, Two-point boundary value problems with eigenvalue parameter contained in the boundary conditions, Proc. Roy. Soc. Edinburgh, Sect. A: Math. 77 (1977), no. 3-4, 293-308. 
[19] N.Yu. Kapustin, E.I. Moiseev, On the basis property in the space $L_{p}$ of systems of eigenfunctions corresponding to two problems with spectral parameter in the boundary condition, Diff. Equ. 36 (2000), no. 10, 1357-1360.

[20] N.Yu. Kapustin, On a spectral problem arising in a mathematical model of torsional vibrations of a rod with pulleys at the ends, Diff. Equ. 41 (2005), no. 10, 1490-1492.

[21] N.B. Kerimov, Z.S. Aliyev, On oscillation properties of the eigenfunctions of a fourth order differential operator, Trans. Acad. Sci. Azerb. Ser. Phys.-Tech. Math. Sci. 25 (2005), no. 4, 63-76.

[22] N.B. Kerimov, Z.S. Aliyev, The oscillation properties of the boundary value problem with spectral parameter in the boundary condition, Trans. Acad. Sci. Azerb. Ser. Phys.Tech. 25 (2005), no. 7, 61-68.

[23] N.B. Kerimov, Z.S. Aliyev, On the basis property of the system of eigenfunctions of a spectral problem with spectral parameter in the boundary condition, Diff. Equ. 43 (2007), no. 7, 905-915.

[24] N.B. Kerimov, Z.S. Aliyev, Agayev E.A. On the oscillation of eigenfunctions of a fourth-order spectral problem, Doklady Math. 85 (2012), no. 3, 355-357.

[25] N.B. Kerimov, R.G. Poladov, Basis properties of the system of eigenfunctions in the Sturm-Liouville problem with a spectral parameter in the boundary conditions, Doklady Math. 85 (2012), no. 1, 8-13.

[26] A.N. Krylov, On some differential equations of mathematical physics having applications to technical problems, GITTL, Moscow-Leningrad, 1950 (in Russian).

[27] E.I. Moiseev, N.Yu. Kapustin, On the singularities of the root space of one spectral problem with a spectral parameter in the boundary condition, Doklady Math. 66 (2002), no. $1,14-18$.

[28] M.A. Naimark, Linear differential operators, Ungar, New York, 1967.

[29] M. Poisson, Mémoire sur la Manière d'experimer les Fonctions par des Séries de quantités periodiques, et sur l'Usage de cette Transformation dans la Résolution de différents Problémes, Journal de l'Ecole Royale Polytechnique, 11 (1820), no. 18, 417489.

[30] E.M. Russakovskii, Operator treatment of boundary problems with spectral parameters entering via polynomials in the boundary conditions, Funct. Anal. Appl. 9 (1975), no. 4, 358-359.

[31] A.N. Tikhonov, A.A. Samarskii, Equations of mathematical physics, Dover, Mineola, 2011.

[32] S.P. Timoshenko, Strength and oscillations of elementary constructions, Nauka, Moscow, 1975 (in Russian).

[33] J. Walter, Regular eigenvalue problems with eigenvalue parameter in the boundary condition, Math. Z. 133 (1973), no. 4, 301-312.

Vuqar A. Mekhrabov

Baku State University, Z. Khalilov str., 23, AZ1148, Baku, Azerbaijan

E-mail address: v-mekhrabov@mail.ru

Received: June 20, 2019; Revised: October 1, 2019; Accepted: October 10, 2019 